\title{
Erythropoiesis-stimulating agents: the present situation
}

\author{
Mario Dicato
}

$\mathrm{E}$ rythropoiesis-stimulating agents (ESAs) have been in clinical use since 1993 in oncology and haematology. It should be stressed today that the licence for marketing by the various regulatory authorities was then given for chemotherapy-induced anaemia with the intent to decrease or avoid blood transfusion. No marketing licence included treatment for fatigue or improvement of quality of life. In clinical trials ESAs were given outside of the label for treatment of anaemia of cancer, due to iron and/or cytokine imbalance like in other anaemias of chronic diseases. ESAs were also administered in order to increase haemoglobin $\mathrm{Hb}$ far beyond normal levels and hence improve cancer treatment with the intent to reduce intra-tumoral hypoxia more favourable to cancerous than to normal cells. This modality was used in chemotherapy as well as in radiotherapy. The results of these studies have been deleterious and discussed at length in multiple publications over the past few years.

The main unfavourable side effects were venous thrombo-embolic events and an increase in mortality [1].

An additional, still not clarified, issue is the supposed presence of erythropoietin (Epo) receptors (EpoR) on cancer cells and the possibility of tumour progression due to Epo. This latter aspect has been investigated with immunehistochemistry techniques using an antibody (C20, Santa Cruz, California) and many authors doubt the specificity of the technique: the test shows the same cellular/tissue pattern in mice whether they are normal or EpoR knock out [2]. It appears that the antibody, not very specific for EpoR measures or heat shock proteins (HSPs), and hence its positivity in tumour samples correlates with poor prognosis not because of Epo or EpoR, but because elevation of HSP is a feature of poor prognosis [2].

A recent Cochrane meta-analysis has shown an increase of venous thrombotic event (VTE) and mortality [1]. This study, however, including outside-of-the-label studies and $20 \%$ of the weight of the negative result is due to one sin-

\footnotetext{
M. Dicato (凶)

Hematology-Oncology

Centre Hospitalier

1210 Luxembourg

e-mail: sec.hematology@chl.lu
}

gle study in which ESA was given with the intent to reach a supernormal $\mathrm{Hb}$ level.

Another meta-analysis on individual patient data with long-term follow-up did not show any negative signal in regards to progression-free survival and overall survival [3].

With all that has been said above, regulatory authorities in the USA, the FDA, and in the European Community, the EMA, have taken steps to give directives for the usage of ESAs (for details see Ref. [4]).

Scientific societies and organisations like the ESMO, ASCO/ASH, EORTC and NCCN have given guidelines taking into account the published data [5].

This is not the place to discuss all the features of these guidelines, but a few points need to be highlighted:

- All marketed ESAs as of now are treated as a single class and, except for dosages, guidelines and recommendations, apply identically to all despite the fact that no headon comparative studies have been done. The same applies for its biosimilars or, in the North American terminology, "biologic follow-on drugs".

- Regarding quality of life (QoL), this issue of the journal publishes two papers on this topic and over the past lengthy discussions have looked at $\mathrm{QoL}$ and fatigue with contradictory results; regulatory authorities have not really taken a stance here. Both the FDA and EMSA, however, include the clinician's evaluation for giving ESAs at a level of $\mathrm{Hb}$ above the standard threshold of around $10 \mathrm{~g}$ depending on the clinical situation of the patient [4].

- For all instances of ESA usage, blood transfusions have come to be considered an option while the initial indication of 1993 was to use ESAs in order to avoid or decrease blood transfusions.

Standard side effects of transfusions are well known regarding infectious problems, immediate immune reactions and fluid overload. With the filtering of blood at the moment of drawing, lung injury has become rare. However, other presumed side effects like late immunity changes, modifications of transfused red blood cell rheology and function after variable storage shelf life, and others are not taken into account. Hence if the two treatments are considered as a possible option, the physician should discuss the risk-benefit of each with the patient and both need to agree on the option to take. 
- The target level of $\mathrm{Hb}$ recommended is the lowest level necessary to avoid transfusion. This is around $10 \mathrm{~g}$ of $\mathrm{Hb}$, meaning that if the patient's $\mathrm{Hb}$ drops due to chemotherapy toxicity, when $10 \mathrm{~g}$ is reached, treatment with either option (ESA or transfusion) is required. If the patient has a low $\mathrm{Hb}$ level, treatment with either option is needed until $10 \mathrm{~g}$ is reached. If ESAs are used, the minimal amount should be given to reach the set goal, being the lowest $\mathrm{Hb}$ level to avoid transfusion.

A well conducted meta-analysis confirmed that ESAs caused an estimated $17 \%$ increase in mortality relative to control during the active study periods in all patients with cancer and a $10 \%$ increase in relation to control in those undergoing chemotherapy. Subgroup analyses like patient characteristics, tumour type and chemotherapy did not show any particular clinically useful predictive risk factor.

One very important aspect that has been stressed by the regulatory authorities is that no ESA is to be given in an anticipated curative setting like adjuvant breast, colon or lung cancer.

Considering all of the above, the only useful recommendation to the clinician for the moment is to follow the guidelines, stressing the fact that ESAs are supportive care drugs that do not improve the underlying neoplastic disease, hence the risk-benefit should determine the therapeutic decision.

\section{References}

1. Bohlius J, Schmidlin K, Brilliant C et al (2009) Recombinant human erythropoiesis stimulating agents and mortality in patients with cancer. Lancet 373:1532-1542

2. Fandrey J, Dicato M (2009) Examining the involvement of erythropoiesis stimulating agents in tumor proliferation (erythropoietin receptors, receptor binding, signal transduction 9, angiogen- esis and venous thromboembolic events. Oncologist 14[Suppl]:34-42

3. Ludwig H, Crawford J, Osterborg A et al (2009) Pooled analysis of individual patient-level data from all randomized, double-blind, placebo-controlled trials of darbepoietin alfa in the treatment of patients with chemotherapy-induced anemia. J Clin Oncol 27:1-10

4. US Food and Drug Administration. FDA drug safety communication: Erythropoiesis-stimulating agents (ESAs): Procrit, Epogen and Aranesp (last viewed 17/5/2011). http://www.fda.gov/Drugs/ DrugSafety/PostmarketDrugSafetyInformationforPatientsandProviders/ucm200297.htm

5. Rizzo JD, Brouwers M, Hurley P et al (2010) American Society of Clinical Oncology/American Society of Hematology clinical practice guideline update on the use of epoietin and darbepoietin in adult patients with cancer. J Clin Oncol 28:49965010 